\title{
EL IMAGINARIO SOCIAL MARÍTIMO BOLIVIANO. UNA EXPLICACIÓN SOCIAL DE LA POLÍTICA EXTERIOR DE BOLIVIA HACIA CHILE
}

\author{
THE BOLIVIAN MARINE SOCIAL IMAGINARY. A SOCIAL EXPLANATION \\ OF THE FOREIGN POLICY OF BOLIVIA TO CHILE AND THE IMPORTANCE \\ OF ITS MARINE ASPIRATION
}

\author{
Jorge Luis Otero* y Pablo Rivas Pardo**
}

\begin{abstract}
Este artículo propone la idea de Imaginario Social Marítimo Boliviano para explicar la Política Exterior de Bolivia hacia Chile desde una perspectiva social. Esto, supuesto por la necesidad de superar la explicación tradicional que se sostiene que Bolivia instrumentaliza su aspiración marítima por causas de asuntos internos. Para ello se hace una discusión teórica acerca del imaginario social y, posteriormente, se analizan ciertos eventos de la relación bilateral y otros sucedidos en Bolivia como forma explicativa de la incidencia de dicho imaginario.
\end{abstract}

Palabras claves: Bolivia, Chile, Imaginario Social, Aspiración Marítima.

This article proposes the idea of Bolivian Maritime Social Imaginary to explain the Bolivian Foreign Policy towards Chile from a social perspective. This based on to necessity to overcome the traditional explanation that Bolivia exploits, due to internal issues, its maritime aspiration. For which, it makes a theoretical discussion on social imaginary. Later, it analyzes some events of the bilateral relationship and others events happened in Bolivia as an explicative way on the incidence of that concept.

Key words: Bolivia, Chile, Social Imaginary, Maritime Aspiration.

\section{Introducción}

La relación bilateral entre Chile y Bolivia tiene como constante la realidad mediterránea boliviana, la que ha llevado a momentos de acercamiento, para buscar eventuales soluciones para una salida marítima soberana o no soberana para Bolivia, y de inestabilidades diplomáticas, cuando esa búsqueda se ve imposibilitada. Esta realidad no es algo nuevo, al contrario, viene desde la década del 20 del siglo XX, cuando se busca internacionalizar la aspiración marítima al ser presentada en la Liga de las Naciones, que buscaba dejar sin efecto el Tratado de Paz y Amistad de 1904. En retrospectiva, esta realidad entre ambos Estados tiene un inicio muy pretérito, brevemente: la Guerra del Pacífico y el Pacto de Tregua fueron en el siglo XIX, la firma del Tratado de Paz y Amistad es de inicios del siglo XX, y la petición de una solución marítima soberana ha llegado hasta el siglo XXI, en consecuencia, el interés nacional entre ambos Estados se ha enfocado en "soberanía e integridad territorial" (González et al. 2016: 70) por sobre otros temas.
Desde la década de los 80 del siglo XX han surgido discusiones y conversaciones entre ambos Estados respecto de la aspiración marítima de Bolivia (Ovando 2015). En la actualidad, el evento más importante y que inició la actual relación bilateral entre ambos Estados es la advertencia de demanda internacional por parte del presidente Morales en la conmemoración del Día del Mar del 23 de marzo de 2011. En palabras de su discurso: "la lucha por nuestra reivindicación marítima, lucha que ha marcado nuestra historia por 132 años, ahora debe incluir otro elemento fundamental: el de acudir ante los tribunales y organismos internacionales, demandando en derecho y en justicia, una salida libre y soberana al océano Pacífico" (Morales 2011: Web). Previo a este anuncio, las relaciones bilaterales seguían patrones regulares, pero se complicaron aún más con la creación, días después del discurso, de la Dirección Estratégica de Reivindicación Marítima (DIREMAR), encargada de "elaborar y presentar la demanda internacional para la reivindicación marítima boliviana" (Bolivia 2011: 02). Vale recordar que Evo Morales asumió

\footnotetext{
* Investigador independiente. Santiago, Chile. Correo electrónico: jotecol@ gmail.com

** Universidad Arturo Prat. Instituto de Estudios Internacionales. Iquique, Chile. Correo electrónico: p.rivas.pardo@ gmail.com
} 
en enero de 2006 la presidencia de Bolivia, casi dos meses antes que lo hiciera Michelle Bachelet en Chile en su primera presidencia. Entre los años 2006 y 2010 la relación bilateral entre ambos Estados fue de crecimiento. Inclusive, se puede sostener que no hay un antecedente mejor que este periodo en alguno de los gobiernos de la Concertación, lo que se explica por la creación de la Agenda de los 13 puntos, la que incluía en los títulos "VI. Tema marítimo (...) VII. Silala y recursos hídricos" (Ministerio de Relaciones Exteriores 2010: 13). Con la llegada de Sebastián Piñera a la presidencia de Chile la relación dio inicialmente continuidad a la Agenda de los 13 puntos. Ejemplo de aquello es la "XXII reunión de mecanismos de consulta" entre ambos subsecretarios de Relaciones Exteriores realizada en julio de 2010 en Bolivia, se consideraron nuevamente los títulos "VI. Tema marítimo (...) VII. Silala y recursos hídricos" (Ministerio de Relaciones Exteriores 2010b: 09). Otros ejemplos son la visita de Morales a la celebración del Bicentenario de la República de Chile en septiembre de 2010, y su posterior viaje al rescate de los 33 mineros de la mina San José en octubre de ese mismo año, ya que había un minero boliviano entre los atrapados.

Sin embargo, la continuidad y buena relación se truncó, presumiblemente, por tres hechos. El primero, fue la captura en el verano de 2011 del exgeneral Sanabria, Jefe de la Policía de Bolivia contra el narcotráfico entre 2007 y 2008 por parte del gobierno de Chile y la DEA, al ser acusado de narcotráfico. Tal operativo no incluyó a las autoridades bolivianas, dejando a Morales con una mala imagen pública, como él mismo señaló: "según algunas informaciones, algunas autoridades de Chile ya sabían, ya hacían seguimiento. Yo me pregunto, si ya sabían, ¿por qué no nos comunicaron oportunamente para dar (a Sanabria) un castigo severo en Bolivia?" (Cornejo 2011: Web). El segundo hecho es el argumento esgrimido por el gobierno de Bolivia, que esperaba una propuesta concreta para un acceso soberano al mar, lo que habría inducido a la demanda en la Corte International de Justicia (CIJ), presumiéndose una inspiración en la demanda que el Perú presentó contra de Chile el año 2008, aún más, cuando el precursor de la demanda marítima del Perú, Manuel Rodríguez Cuadros, fue Embajador del Perú en Bolivia entre febrero de 2010 y agosto de 2011. El tercer hecho, según Evo Morales, es que durante el primer gobierno del presidente Piñera se habría suspendido la reunión de Consultas Políticas prevista para octubre de 2010 (Cádiz 2016: Web).

Finalmente, el gobierno de Bolivia interpuso la demanda en la CIJ contra Chile en abril de 2013, en la que sostiene, resumidamente, que "Chile tiene la obligación de negociar con Bolivia para llegar a un acuerdo que conceda a Bolivia un acceso soberano al océano Pacífico" (Bolivia 2013: 20). Posteriormente, la degradación de la relación bilateral fue en aumento, y el 23 de marzo de 2016 el presidente Morales anunció que demandaría a Chile por los usos del río Silala, a lo que el gobierno de Chile decidió adelantarse, presentando su demanda pertinente a ese tema en junio de 2016, en la que se manifiesta, entre otras peticiones a la CIJ, que "con arreglo a la norma de utilización equitativa y razonable, Chile tiene derecho a su uso corriente de las aguas del río Silala" (Chile 2016: 11).

En resumen, la buena relación entre los años 2006 y 2011 se dio en un marco donde Bolivia creía que su aspiración marítima era más viable, pero tras el episodio del exgeneral Zanabria, agotada la espera de una propuesta concreta y la discontinuidad de la Reunión de Consultas Políticas, se terminó el diálogo bilateral. La siguiente etapa es de confrontación verbal donde dominaría el anuncio y luego interposición de la demanda judicial internacional de Bolivia contra Chile. En consecuencia y en vista de la extensión del tiempo, la intermitencia de esta relación bilateral, y por sobre todo, la importancia de la aspiración marítima boliviana y el cambio de la Política Exterior de Evo Morales hacia Chile, desde el diálogo a la demanda, se propone como pregunta de este artículo:

\section{¿Qué explica la constante aspiración marítima de Bolivia en su Política Exterior hacia Chile?}

La respuesta es sencilla. Esta determinación se debe-como lo explica la retórica del presidente Evo Morales-a que Bolivia, siendo República, tuvo acceso soberano al mar. Como antecedente histórico, el límite internacional entre ambos Estados era el paralelo $24^{\circ}$, según lo indica el Tratado de Límites de 1866 (Chile 1866: Web) y que tras el Tratado de Paz y Amistad de 1904, donde Bolivia cede la provincia de Antofagasta a perpetuidad a Chile tras la Guerra del Pacífico, ha buscado restituir su cualidad marítima. Sin embargo, tal respuesta es 
complementada con un analisis social, es decir, la pregunta se responde desde una perspectiva social en cuanto a la permanente búsqueda de aspiración marítima.

Para responder la pregunta de este artículo, este se divide en dos secciones. Primero se presenta la teoría que sustenta la idea de "Imaginario Social Marítimo Boliviano" que explica cómo las sociedades crean y recrean sus interpretaciones de la realidad. La segunda sección es el análisis de cómo se ha construido este Imaginario Social, el que se subdivide en la "Desestimación de la Explicación Tradicional" y en el "Inicio y Desarrollo del Imaginario Social Marítimo Boliviano". Por último, en las conclusiones se responde la pregunta y finaliza con una reflexión de la relación política estructural entre ambos Estados.

\section{Cornelio Castoriadis y el Imaginario Social}

Para analizar los eventos, hechos y sucesos que permiten afirmar que la Política Exterior del gobierno de Evo Morales hacia Chile es consecuencia de una construcción histórico-social de Bolivia y los bolivianos, se emplea el concepto "Imaginario Social Marítimo Boliviano", el que inicia su sustento teórico en las ideas expuestas en el artículo "Bolivia y Chile: crisis, gas, mar e imaginarios sociales" de Andrés Aliaga. El autor explica un fenómeno ocurrido en la crisis del gas en Bolivia el año 2003, momento en que las protestas sociales y comunitarias provocaron la renuncia del presidente Sánchez de Lozada. Aliaga indica que "en este plano de activación social es cuando surge el tema, con más fuerza por cierto, de la reivindicación marítima, lo cual desde mi perspectiva surge producto de los fuertes remezones que ha vivido recientemente esta sociedad, lo que fue combinado con un juego histórico, político y mediático" (2006: 15). Esta cita indica que el conflicto social ocurrido hizo que se manifestaran algunos elementos latentes que surgen en momentos de catarsis, y en este caso fue la aspiración marítima. En consecuencia, una explicación histórico-social de la Política Exterior de Morales no puede estar alejado de lo que ocurre con los bolivianos y la creación imaginaria social del mar.

En el mismo artículo se define el imaginario social como "una manera compartida por grupos de personas de representarse mentalmente el espacio y tiempo. Algo así como un imaginar o idear socialmente, en donde se comparten, en una modalidad simbólica, formas y contenidos" (Baeza 2000: 09; en Aliaga 2006: 11). Posteriormente, en relación con el mar, a lo social e imaginario Aliaga cita a Cornelio Castoriadis para explicar que la existencia de una "significación social imaginaria, son necesarios unos significantes colectivamente disponibles" (Castoriadis 1999: 251; en Aliaga 2006: 12) poniendo como ejemplo la conmemoración del Día del Mar, acto oficial del Estado donde participan políticos, civiles y militares. Esto último se presenta como un buen caso de grupos de personas que conmemoran, evocan y recrean símbolos y contenidos referidos a la aspiración marítima.

Castoriadis desarrolló una extensa y compleja reflexión acerca del imaginario social, que no puede, según el autor, ser explicado ni por la lógica ni la racionalidad (Castoriadis 1997) y donde plantea una nueva ontología particular del ser de lo histórico-social, denominada magma (Cristiano 2012), y definido como "el modo de organización de una diversidad no susceptible de ser reunida en un conjunto, ejemplificada por lo social, lo imaginario o lo inconsciente" (Castoriadis 2013: 293). Está compuesto por una trama entrelazada de significaciones imaginarias sociales que escapan de lo racional pero que "empapan, orientan y dirigen toda la vida de la sociedad considerada y a los individuos concretos que corporalmente la constituyen y que, por así decirlo, la animan" (Castoriadis 2005: 68). Las significaciones imaginarias sociales que componen el magma y, al mismo tiempo, constituyen el imaginario social, se encuentran materializadas en las instituciones de la sociedad, estableciendo su unidad social -cohesión interna-y su propio sistema de interpretación del mundo (Castoriadis 2008), es decir, su identidad (Castoriadis 2005). La institución de la sociedad no es más que la suma de instituciones particulares que forman un todo cohesionado y organizado a su interior, que finalmente direcciona la vida del individuo social.

Las significaciones imaginarias sociales crean el sentido de una sociedad, dan vida y fundamentan las instituciones, y se dividen en significaciones centrales y significaciones segundas, siendo las últimas las que dan sentido a las primeras. Por ejemplo, Dios es una significación imaginaria social central que no significa lo mismo para un cristiano, musulmán o un judío, estas diferencias de significados son determinadas por las 
significaciones segundas, que se expresan en diferentes símbolos y rituales que, asociadas a las significaciones centrales, le dan contenido y significado. Siguiendo el mismo ejemplo de Dios, los distintos rituales religiosos, la cruz de Cristo o la estrella de David, entre otros, son elementos que le dan contenido y significado tanto al Dios de los judíos como al Dios de los cristianos. Tanto para unos como para otros, sin las significaciones segundas, las centrales carecerían de cualquier sentido. Las significaciones imaginarias le dan vida y fundamento a las instituciones, y son creadas por un poder denominado instituyente.

En ese sentido, el poder instituyente surge de la fuerza creadora de la propia sociedad, es decir, del colectivo anónimo (Cristiano 2012). Por tanto, la acción instituyente pasa a ser un cimiento fundamental para la formación de una identidad social de unidad colectiva, sin este, el magma de significaciones imaginarias sociales sería estéril y estático, incapaz de tener vida continua en la sociedad y, por tanto, carente de imaginario social. Complementando esta idea, el imaginario social comprende "tanto las prácticas como las representaciones que se refieren a las identidades de los miembros de una comunidad sociopolítica; esto es, a los modos de pertenencia, normas comunes y aspiraciones, asignación de significado a acontecimientos que se consideran cruciales, y narrativas diversas" (Girola 2007: 49). El resultado final de esto es la creación de una institución, que son las "normas, valores, lenguaje, instrumentos, procedimientos y método para tratar las cosas y hacer las cosas" (Castoriadis 1986: 11). Así también, las significaciones imaginarias sociales se constituyen por "los espíritus, los dioses, Dios, la polis, el ciudadano, la nación, el Estado, el partido, la comodidad, el dinero, el capital, la tasa de interés, el tabú, la virtud, el pecado, etcétera" (Castoriadis 1986: 12), es decir, los elementos que componen la institucionalidad y la interacción entre las personas. Estas significaciones se encuentran materializadas en las instituciones de la sociedad, determinando lo que es real y tiene sentido de lo que no, estableciendo su unidad social y cohesión interna, y su propio sistema de interpretación del mundo (Castoriadis 2008). El imaginario social, así como también las significaciones imaginarias sociales, no puede ser estudiado analíticamente como concepto, "es decir, por medio de categorías y de operaciones con conjuntos" (Castoriadis 2005: 73), sino que desentrelazando las diferentes significaciones imaginarias que se encuentran impregnadas en la sociedad y sus instituciones, y que explican la identidad de la propia sociedad, desde un contexto histórico-social.

El Imaginario Social Marítimo Boliviano (ISMB) propuesto en este artículo engloba la construcción del sentido de la aspiración marítima boliviana, y a su vez explica la Política Exterior de Bolivia. Entonces, este ISMB se construye por un imaginario social, el que incluye símbolos, formas, normas, valores, creaciones y contenidos que son comunes, colectivos, disponibles como un acto reflejo y que se estampan en diferentes instituciones. Su disponibilidad se da en una comunión de espacio y tiempo que permite imaginar, crear o idear socialmente los símbolos y sus contenidos. Debido a que lo histórico y lo social es indivisible, la clase política no está exenta de ser parte de este imaginario social, por lo que absorbe y replica su contenido, teniendo como consecuencia las acciones instituyentes transmitidas por el magma que son el canal de las significaciones imaginarias.

\section{El Imaginario Social Marítimo Boliviano}

\section{Desestimación de una explicación tradicional}

Se entiende por una explicación tradicional el hecho que en Bolivia un gobierno apela a su aspiración marítima como forma de salir de sus problemas internos, resulta una afirmación incompleta. Si bien la instrumentalización puede ser útil para aunar voluntades al interior de un país, coalición política o aliviar una crisis interna, no existe necesariamente una causalidad en la correlación: crisis e instrumentalización. En cambio, sí hay hechos puntuales que apelan a una exacerbada beligerancia por parte del presidente Morales respecto del tema, pero es un aspecto que no se aborda en este artículo.

La explicación tradicional tiende a afirmar, entre otras explicaciones, que la política boliviana hace una instrumentalización de la aspiración marítima por una debilidad electoral de sus gobiernos, de sus instituciones, y del sistema de partidos políticos. Leyton (2005) sostiene aquello, empero, el problema metodológico de tal afirmación es que expone las cualidades en abstracto sin explicar la causalidad, es decir, la correlación de datos expuestos no implica causalidad, esta debe ser demostrada con ejemplos 
claros y eso no es cumplido. De manera simple, la causalidad estaría explicada si hubieran repetitivos casos de instrumentalización con evidencia de apoyo electoral, aumento de aprobación gubernamental, presidencial, o por lo menos, que sea real en un momento de crisis. De manera totalmente contraria, Coloma (2012) señala que no existen coincidencias de baja popularidad y un uso instrumental de la aspiración marítima en los casos que él analiza. Como primer ejemplo, el anuncio de demanda del presidente Morales se hizo en un contexto ausente de crisis social o política; otro ejemplo es el reclamo público hecho por el expresidente Meza el 2004, momento en que su aprobación era ya de $78 \%$, es decir, no había problema de respaldo ciudadano; de manera similar, cuando su popularidad bajó a 47\% el 2005 él no elaboró una política agresiva en relación con el tema marítimo; por último, cuando Banzer tuvo 32\% de aprobación durante el 2000, su proximidad con el presidente Lagos fue muy buena, es decir, no instrumentalizó el tema marítimo para así subir su aprobación. En otra publicación de Coloma (2013) se señala que las reformas políticas y económicas que afectan la estabilidad del Estado de Bolivia en las últimas tres décadas imposibilitan indicar causalidad entre crisis internas e instrumentalización de la aspiración marítima. Posteriormente afirma que no existe causalidad entre bajos índices de popularidad y alta conflictividad con ofensivas gubernamentales contra el Estado de Chile o a favor de su aspiración marítima. Dando sustento a lo que plantea Coloma, el expresidente Sánchez de Lozada (2002-2003) renunció en su segundo mandato en medio de una alta crisis social, momento en que él tenía una buena relación con el expresidente Lagos y la intención de exportar gas por Chile (Pérez 2004). Otra situación similar fue el intento de destitución, desde la denominada Media Luna, del presidente Morales el 2008, que no desembocó en una política hostil hacia Chile, tampoco en la instrumentalización del tema marítimo para salir del impasse. Un dato anexo es que la gravedad del asunto llevó a que UNASUR publicara una declaración de apoyo al Presidente y rechazando un intento de destitución civil, cuando la presidencia pro tempore correspondía en ese momento a Michelle Bachelet (Rivas y Sepúlveda 2011).

Los datos demuestran que la explicación tradicional: a baja aprobación política del poder ejecutivo o alta crisis política o social, desemboca en una instrumentalización del tema marítimo, no es evidente. Además, no es capaz de explicar la compleja relación entre ambos Estados. Así también, no se encuentran casos respecto de crisis de gobernabilidad en Bolivia o de baja popularidad de un Presidente que desemboquen en una política hostil hacia Chile o que manipule el asunto marítimo para salir del statu quo. En cambio, sí es posible ver hechos aislados, como la beligerancia mostrada por el presidente Morales al señalar que eran chilenos quienes promovían las protestas en Potosí en julio de 2015 (Infobae 2015), pero no una estructura comunicacional dedicada a eso en momentos de crisis.

\section{Inicio y desarrollo del Imaginario Social Marítimo Boliviano}

\section{A. El origen de lo social y marítimo}

Cronológicamente, y desde la perspectiva del poder político, el primer evento importante tras el Tratado de 1904 es la acción política boliviana ante la Liga de las Naciones, entre 1920 y 1921, donde buscó lo que el gobierno boliviano definía como "la reversión de la injusta situación creada por el Tratado de 1904" (DIREMAR 2012b: 06). En este mismo contexto, solo un par de años después se produce el primer acto nacional de carácter cívico, que fue la promulgación de la ley "Día del Litoral" el 23 de marzo de 1922, que con el tiempo devino en el Día del Mar. Tal ley indica en su Artículo Único: "Declárase 'Día del Litoral' el 23 de marzo, aniversario del heroico sacrificio de Abaroa y de la gloriosa actuación de Ladislao Cabrera y sus compañeros en la acción de Calama, debiendo tributarse, con tal motivo, en toda la República, homenajes que tiendan a estimular en el pueblo el culto a sus héroes y el anhelo de reivindicar su patrimonio marítimo" (Bolivia 1922: Web).

De estos hechos y analizado desde la clase política, esta hizo ante la Liga de las Naciones una apertura de su aspiración marítima a nivel internacional, que luego, con la promulgación de la ley del "Día del Mar", lo instala a nivel nacional, asunto que puede ser más importante para apoyar un imaginario social de lo marítimo. Un segundo elemento de la ley es la instalación de los defensores de Calama, ambos civiles, como la principal gesta durante la guerra, que con los años instaló a Eduardo Abaroa como su principal héroe, recordado por su expresión "rendirme yo ¡qué se rinda su abuela... Carajo!". 
(DIREMAR 2011: 24) frente al ofrecimiento de rendición. El tercer elemento es que cada 23 de marzo se conmemora y celebra la "reivindicación de su patrimonio marítimo" a nivel nacional, que a primera vista no implica textualmente el volver al mar de manera soberana, sino que mantener una cultura respecto de la memoria del Litoral. Es paradójico que en 1952, al trasladarse los restos de Eduardo Abaroa, enterrado en Calama hasta ese momento, desde Arica a la frontera con Bolivia, fuese escoltado por el Ejército de Chile, inaugurandóse en $\mathrm{La} \mathrm{Paz} \mathrm{un} \mathrm{monumento} \mathrm{en} \mathrm{su} \mathrm{honor} \mathrm{en}$ la Plaza que lleva su nombre, donde cada año se hace el acto central del gobierno para el Día del Mar (Marín 2007).

En consecuencia, Bolivia comenzó su reconstitución de memoria muy tardíamente, haciendo una lectura común para los países de América Latina durante el siglo XIX, compuesta de "virtudes patrióticas, amor y sacrificio hacia y por la patria, profundas convicciones de que la historia se construye a través de actos heroicos, alto sentido del valor militar" (Cavieres 2013: 31). Lo anterior trae como consecuencia la creación de espacios para que esas virtudes tengan un centro referencial material, no es suficiente lo intangible "sino también de espacios y monumentos consagratorios relevantes de la vida civil, de rituales y símbolos, y de galerías de héroes forjadores de la patria y defensores de esta en momentos de conflictos" (Cavieres 2013: 33), siendo el ejemplo más claro la Plaza Abaroa. La instalación del Día del Litoral debe ser interpretada como el día en que un grupo de personas, es decir, una comunidad nacional, puede imaginar, idear y recrear un contenido épico y virtuoso. Así también, y con el devenir de los años, estos valores se han personificado en Eduardo Abaroa, que pasa a ser un símbolo del litoral, se personifica, con el tiempo, en un símbolo de la aspiración marítima boliviana. Vale destacar que al declararse este día por parte del poder político, el deseo de tener un acceso soberano al mar se transforma en algo propio de la élite que le fue traspasado a la sociedad completa, formando el mar en un significate colectivo.

\section{B. La construcción del imaginario actual}

La relación bilateral actual no tiene rango diplomático, ya que en 1963 el gobierno de Bolivia retiró su embajador por una disputa relativa a los usos del río Lauca, que en su momento implicaron "violentas manifestaciones antichilenas en Bolivia" (González et al. 2016: 61). Posteriormente, las relaciones fueron reestablecidas temporalmente entre 1975 y 1978 , en el marco del proceso de negociación de una fórmula de acceso soberano al mar con canje territorial, conocida como Charaña.

$\mathrm{El}$ origen de ese episodio se produjo entre los regímenes militares de los generales Hugo Bánzer y Augusto Pinochet, tras una conversación en Brasilia durante la asunción presidencial de Gaisel en marzo de 1974. En testimonio de Bánzer, según un documental de DIREMAR: "allí yo le dije al presidente Pinochet que ya que los políticos o los civiles no habían podido hasta esa fecha avanzar nada en nuestro problema marítimo (sic) que tenía todo el deseo que dos militares pudieran entenderse" (DIREMAR 2013: Web). En esa etapa se restablecieron las relaciones diplomáticas durante todo el período de intercambios, desde febrero de 1975 con el llamado "Abrazo de Charaña", hasta la ruptura declarada por Bolivia en marzo de 1978, desahuciando el proceso.

$\mathrm{Al}$ año siguiente se conmemoró el centenario de la Guerra del Pacífico, año en que existió una movilización comunicacional en Bolivia, de hecho, ese año fue declarado como el "año del centenario del litoral cautivo", expresión que fue incluida en todos los documentos oficiales del Estado (De la Calle 1979: Web). El 14 de febrero de 1979 a las 12:00 horas sonaron las sirenas y campanas en todo el país paralizando las actividades en memoria al desembarco en Antofagasta. Otro acto ocurrido ese mismo día fue la lectura en acto público del Jefe de Estado, general David Padilla, de una proclama, diciendo: "Compatriotas, juremos por Dios, la Patria y la memoria de nuestros héroes, que jamás renunciaremos a recuperar nuestro mar ¿Lo Juraís?" (DIREMAR 2013: Web).

En la política internacional, el tema fue presentado en la OEA, cuya Asamblea General adoptaría una resolución en términos de que era de "interés hemisférico permanente encontrar una solución equitativa por la cual Bolivia obtenga acceso soberano y útil al Océano Pacífico" (Milet 2004: 37). Luego, desde 1979 hasta 1989 se emitieron "once resoluciones de la OEA sobre la cuestión marítima" (DIREMAR 2012: 21), las que fueron modificando el lenguaje progresivamente. A esto se suman las acciones multilaterales que hicieron distintos gobiernos de Bolivia en las Conferencias de los Países no Alineados, el Parlamento Latinoamericano y la 
Asamblea General de Naciones Unidas (Figueroa 2007).

Por su parte, la sociedad civil ha respondido a estas acciones de la clase política, como son la fundación del "Comité Cívico Pro Mar Boliviano" en febrero de 1974, por parte de Gaby de la Reza, más conocida como Gaby del Mar, cuyas funciones se extienden hasta hoy. El objetivo de esta institución es "incentivar el civismo y forjar una conciencia marítima en la ciudadanía, basada en la reflexión histórica de recuperar la cualidad marítima de Bolivia" (DIREMAR 2011c: 14). Otro evento fue la fundación de "El Museo del Litoral", por parte del municipio de La Paz en julio de 1978, donde se exponen mapas de lo que fue el Litoral boliviano, pinturas de buques bolivianos y antecedentes de la Guerra del Pacífico, enalteciendo la figura de Abaroa. Como resultado se crea la sensación que "su desarrollo efectivo está ligado a la satisfacción urgente de esta necesidad nacional" (Valencia 1982: 535), es decir, que su desarrollo país depende de un acceso al mar, en otras palabras, "el problema de la mediterraneidad boliviana sigue siendo central en la historia del conflicto y en las necesidades de la nación boliviana" (Cavieres y Cajías 2007: 162).

Estos eventos son sustanciales para la comprensión de la política exterior de Bolivia, no solo con Chile, sino también con otros países, mandatarios y en lo multilateral. Si en 1922 se creó el día del Litoral y se comenzó a construir un ideario nacional, en la década de los 70, con la negociación BánzerPinochet, la conmemoración del Centenario de la Guerra del Pacífico y la acción internacional, se inicia la estructura política bilateral actual, donde la variable acceso soberano al mar se transforma en una variable monocausal entre Bolivia y Chile. Con esto, el Imaginario Social respecto del mar se vuelve irreversible, tanto para la sociedad civil como para la clase política, produciéndose una indivisión entre ambas partes, es decir y como señala Castoriadis, hay una mutua influencia entre lo histórico-social. El concepto magma que utiliza Castoriadis explica cómo con los años el asunto ha sido perenne y ha adquirido su propias normas, como fue la incorporación de la aspiración marítima en la Constitución Política de 2009; de valores, al tener un talante épico de juramento nacional; en el lenguaje, donde el mar aparece como una palabra idílica; y en la construcción de la historia como son los casos del Pro Mar y del Museo del Litoral, que a su vez es constructora de la sociedad.
Como resultado, sucesivos gobiernos de Bolivia han propuesto tanto la revisión del Tratado de 1904 como su denuncia. Además, han diversificado el tema en organismos multilaterales, con Estados amigos, y han propuesto mediaciones y negociaciones directas y se ha acercado al Perú (Francisco 2009: 60-61). En consecuencia, no hay una claridad en la definición del problema, la solución del mismo y su respectivo mecanismo, por parte de los sucesivos gobiernos de Bolivia.

\section{Algunas interpretaciones bolivianas}

Tempranamente parte de la historiografía boliviana ha interpretado la firma del Tratado de 1904 como un hecho adverso. En los años 1920 se interpretaba como que Bolivia "no tuvo más remedio que suscribir el 20 de octubre de 1904 el tratado hoy vigente" (Arguedas 1922: 408-409), interpretación que con el tiempo se ha vuelto a exponer en ciertas circunstancias. La DIREMAR ha llevado este enfoque a su máxima expresión, explicando que la firma del Tratado fue "después de 25 años de ocupación militar de territorio boliviano, la intervención total de sus aduanas, puertos y de su comercio, además de la amenaza de volver al estado de guerra y reiniciar las hostilidades militares" (DIREMAR 2011: 14). También que el Tratado "no fue fruto de una negociación libre y consentida por Bolivia, sino que se impuso por medio de la fuerza" (DIREMAR 2011b: 09). Tal construcción, repetitiva, difundida y excluyente, obvia que al momento de la firma Bolivia tenía la necesidad de exportar su gran industria minera, y especialmente la del estaño, por lo que el mismo Tratado le da garantías portuarias para el movimiento de mercadería. Así también, que Arica, el puerto de acceso más directo para Bolivia, tendría regalías que históricamente nunca había tenido, ya que tal puerto no fue parte del territorio boliviano, ni como República ni como Colonia. Sin embargo, lo historico-social nos indica que el recuerdo del Tratado de 1904 se repite como una rapsodia sagrada, irreflexiva y muy emocional.

Otro elemento importante de analizar es la creación de los héroes patrios de la Guerra del Pacífico. Como se expuso antes, en 1922 se recordaron a los defensores de Calama, dos civiles que con el tiempo han sido los más venerados, pero no son los únicos, también hay dos menores, una es Genoveva Ríos, que tras el desembarco en Antofagasta rescató la bandera de Bolivia de la prefectura: "la niña dobló 
la enseña patria, escondiéndola entre sus ropas, mientras sigilosamente abandonaba la Prefectura para reunirse con sus padres y buscar refugio" (DIREMAR 2011: 25). El otro es un niño, llamado Juan Pinto, más conocido como Juancito Pinto, perteneciente al Regimiento Colorados, que falleció en la Batalla del Alto de la Alianza o de Tacna, en mayo de 1880, "ingresando al combate en medio de los feroces estruendos de cañones y ráfagas de la fusilería chilena" (DIREMAR 2011: 25). Lo extraño, curioso y, quizás, exclusivo de la memoria boliviana acerca de la Guerra del Pacífico es que en el imaginario social común de los bolivianos se recuerda a dos civiles y dos niños, en vez de los comandantes militares. Para Bolivia la guerra duró algo más de un año. Tras la batalla donde hubo más muertos y combatió su regimiento más tradicional, Bolivia recuerda a un niño y no a su comandante, oficiales, suboficiales o soldados. Se ha preferido por la simbología de dos niños y dos civiles, lo que se puede entender como una instrumentalización para que la aspiración marítima sea más amplia que el sector militar o nacionalista, e involucre a civiles como Abaroa y Cabrera, y cause la simpatía y atracción por los niños. La creación de este contenido puede ser ejemplo que otros contenidos de la aspiración boliviana también puede obedecer a una práctica selectiva y arbitraria, donde su misión final no es informar, sino que concientizar.

En cuanto a los emblemas patrios, el expresidente Carlos Meza modificó el escudo nacional, incorporando una estrella adicional, la que simboliza el "Departamento del Litoral" (Mesa 2004), que también cuenta con su propia bandera y escudo. De manera similar, pero muchos años antes fue creada la "Bandera de la Reivindicación Marítima" (Ovando, 1966), la que ha alcanzado gran popularidad con el actual gobierno de Bolivia, el que también ha publicado varios libros como el Libro del Mar (DIREMAR 2014).

\section{Conclusiones}

Como señala la cita de González en la Introducción, la relación entre Chile y Bolivia está determinada por la "soberanía e integridad territorial", las que inciden en la relación en su totalidad. Esto, desde la perspectiva de Castoriadis, ha creado una relación historico-social que pone a la aspiración marítima como lo central entre ambos Estados y sociedades. Para Bolivia, esto ha creado un Imaginario Social que prontamente cumplirá 100 años, desde la acción ante la Liga de las Naciones. Entonces, frente a la pregunta ¿Qué explica la constante aspiración marítima de Bolivia en su Política Exterior hacia Chile? Se responde que:

Primero, tal asunto no es coyuntural, por el contrario, es estructural. El Imaginario Social Marítimo Boliviano es parte del ser social nacional, compuesto por significantes colectivos que transforman tal Imaginario en algo constante y real.

Segundo, la construcción de los significantes colectivos del Imaginario Social Marítimo Boliviano datan de los años 20 del siglo XX, tanto por la acción en la Liga de las Naciones como por la institucionalización del Día del Litoral/Mar. Posteriormente se fueron profundizando con la innovación de llevar la aspiración marítima a la OEA y a otras instancias multilaterales, lo que se profundizó aún más con la innovación de demandar al Estado de Chile ante la Corte Internacional de Justicia. Vale destacar que la acción ante la OEA ha sido un punto de no retorno, ya que tras ello Bolivia no se ha limitado únicamente a lo bilateral.

Tercero, un primer significante colectivo son los héroes de la Guerra del Pacífico, por un lado Abaroa y Cabrera, ambos civiles, y por otro los niños Genoveva y Juancito. Esto resulta extraño, ya que en la guerra combatió el Regimiento No 1 "Los Colorados de Bolivia", que es la unidad militar más tradicional de aquel país, pero sus comandantes y combatientes no figuran al nivel heroico de estos cuatro personajes. $\mathrm{La}$ ausencia de militares puede suponer una manipulación interna de no incluir a uniformados, o de manera contraria, que no existió una real participación de ese país con su Ejército en la guerra. Un segundo significante colectivo es que la aspiración marítima se asume como una causa justa, por ejemplo, el año del Centenario de la Guerra del Pacífico se denominó como el "año del centenario del litoral cautivo", siendo la palabra cautivo la que simboliza la justicia de la aspiración marítima para terminar con un tratado injusto de una guerra injusta. Además, esto se llevó a nivel nacional, teniendo respuestas como el Comité Cívico Pro Mar y el Museo del Litoral. Un tercer significante es la propaganda 
hecha por el actual gobierno, como son el Libro del Mar, la bandera y escudo del Litoral y la bandera de la Reivindicación Marítima.

A modo de reflexión final, el presidente Morales usó su poder instituyente para crear la DIREMAR y difundir su aspiración marítima a nivel nacional e internacional, dejando el diálogo por la demanda. Con esto, ha replicado, perpetuado y agrandado el discurso de aspiración marítima, con ello el Imaginario Social Marítimo Boliviano se consolidó. Una de las consecuencias sociales es que la amplificación de los símbolos, formas, contenidos, es decir, la interpretación del mundo y creación de un propio cosmos argumental ha creado una oposición total a Chile. Entonces, la aspiración marítima es un asunto social que forma y reproduce un sentido cultural, hace compleja la relación, ya que la solución marítima soberana determina la existencia o no de una buena relación bilateral. Así también, el costo de tener una eventual e hipotética buena relación entre ambos Estados es a costa del territorio de Chile, en razón del Imaginario Social que se ha creado en Bolivia.

\section{Referencias Citadas}

Aliaga, F

2006 "Bolivia y Chile: crisis, gas, mar e imaginarios sociales". Revista Electrónica Ciencias Sociales Online, Universidad de Viña del Mar, marzo 2006, Vol. III, Nº 1, pp. 1-18.

Arguedas, A.

1922 Últimos Sucesos 1900-1921, Historia General de Bolivia, Archivo y Biblioteca Nacional de Bolivia, pp. 407-427.

Cádiz, P.

2016 Choquehuanca responde a Embajador Gaspar: Quien posterga el diálogo no es Bolivia, es Chile. Tele 13 Radio, 6 de enero de 2016, Web: http://www.t13.cl/noticia/politica/ choquehuanca-responde-embajador-gaspar-quien-postergadialogo-no-es-bolivia-es-chile

Cavieres, E. y Cajías, F.

2008 "El gran quiebre: la Guerra del Pacífico. Sus contextos y sus efectos". Chile-Bolivia, Bolivia-Chile: 1820-1930. Desarrollos Políticos, Económicos y Culturales. Pontificia Universidad Católica de Valparaíso y Universidad Mayor de San Andrés, pp. 125-162.

Cavieres, E.

2013 "Región y nación. relaciones vecinales, historia e integración. Desafíos pendientes y tareas inconclusas". Revista Diálogo Andino, № 42, pp. 31-40.

Castoriadis, C.

1986 "El campo de los social histórico". Revista Estudios. Filosofía, Historia, Letras, № 4, primavera de 1986, ITAM, México, pp. 7-25.

Castoriadis, C.

1997 "Capítulo VII: Antropología, filosofía, política”. En El Avance de la Insignificancia. Argentina. EUDEBA, pp. 131-154.

Castoriadis, $\mathrm{C}$.

2005 "Lo imaginario: la creación en el dominio historicosocial”. En Los dominios del hombre. Las encrucijadas del laberinto. España, Gedisa Editorial, pp. 64-77.

Castoriadis, C.

2008 "Reflexiones sobre el racismo". En El Mundo Fragmentado, La Plata, Terramar, pp. 29-44.

Castoriadis, C.

2013 "Segunda parte. El imaginario social y la institución". En La institución imaginaria de la sociedad, Buenos Aires, Tusquets, pp. 269-529.
Cristiano, J.

2012 "II. La teoría de la sociedad de Castoriadis". Lo social como institución imaginaria. Castoriadis y la Teoría Sociológica. Argentina. Eduvim. Edición Kindle, pp. 164-338.

Coloma, C.

2012 "Las relaciones entre Chile y Bolivia tras el anuncio del gobierno boliviano de demandar a Chile ante un tribunal internacional". Revista Política y Estrategia, № 119, enerojunio, pp. 131-169.

Coloma, C.

2013 "La estabilidad política en Bolivia y su relación con la demanda marítima”. Revista Política y Estrategia, $\mathrm{N}^{\circ} 122$, julio-diciembre, pp. 15-58.

Cornejo, $\mathrm{H}$.

2011 "Morales reclama a Chile por no avisarle del caso de su ex jefe antinarcóticos". Diario Panamá América, 25 de junio de 2011, Web: www.panamaamerica.com.pa/content/ morales-reclama-chile-por-no-avisarle-del-caso-de-suexjefe-antinarcóticos.

De la Calle, A.

1979 "Perú y Bolivia conmemoran el centenario de su guerra con Chile". Diario El País, Internacional, Archivo electrónico, Web: http://elpais.com/diario/1979/02/14/ internacional/287794809_850215.html

DIREMAR

2011 DIREMAR. Revista Informativa. Año 1, No 1, Julio de 2011, Web: http://www.diremar.gob.bo/node/146

DIREMAR

2011b DIREMAR. Revista Informativa. Año 1, No 3, Septiembre de 2011, Web: http://www.diremar.gob.bo/ node/146

DIREMAR

2012 Mar para Bolivia. Revista Informativa, No 1, Febrero de 2012, Web: http://www.diremar.gob.bo/node/146

\section{DIREMAR}

2012b Mar para Bolivia. Revista Informativa, $N^{\circ} 6$, Diciembre de 2012, Web: http://www.diremar.gob.bo/node/146

DIREMAR

2013 Documental: El mar de Bolivia, Web: https://www. youtube.com/watch? $\mathrm{v}=\mathrm{og}$ XOMBAhPIU\& $\mathrm{t}=2 \mathrm{~s}$

DIREMAR

2014 El Libro del Mar, Web: http://www.diremar.gob.bo/sites/ default/files/LIBRO\%20DEL\%20MAR\%20BILINGUE.pdf 
Figueroa, U.

2007 La demanda marítima boliviana en los foros internacionales. RIL Editores, Santiago de Chile.

Francisco, M.

2009 "La cuestión marítima en la Política Exterior de Chile y Bolivia". En Revista Diplomacia, № 118, enero-marzo, Santiago, pp. 47-69.

Girola, L.

2007 "Imaginarios socioculturales de la modernidad. Aportaciones recientes y dimensiones de análisis para la construcción de una agenda de investigación". Revista Sociológica, Año 22, No 64, mayo-agosto, 2007, pp. 45-76.

González, S.; Ross, C.; Ovando, C.

2016 "La cuestión del río Lauca" desde la perspectiva multiescalar: ¿un juego de suma cero de las diplomacias boliviana y chilena? En Revista Diálogo Andino, No 51, pp. 57-72.

Infobae

2015 Desconfianza: Evo Morales cree que las protestas en Potosí son promovidas desde Chile. Política, Web: https:// www.infobae.com/2015/07/20/1742813-desconfianza-evomorales-cree-que-las-protestas-potosi-son-promovidas-chile/

Leyton, C.

2005 "Instrumentalización de conflictos históricos: el caso chileno-boliviano". Revista Política y Estrategia, No 97, marzo 2005, pp. 9-26.

Mesa, C.

2004 Decreto Supremo No 27630 del 21 julio 2004. Símbolos Patrios. En Derechoteca Bolivia, Web: http://www.derechoteca. com/gacetabolivia/decreto-supremo-27630-del-21-julio-2004/

Ministerio de Relaciones Exteriores de Chile

2010 "Vínculos Vecinales prioritarios". En Hitos de la política exterior, durante el gobierno de la Presidenta Michelle Bachelet, Web: www.minrel.gob.cl/hitos-dela-politica-exterior-publicacion-sobre-los-principales/ minrel/2009-12-30/083503.html

Ministerio de Relaciones Exteriores de Chile

2010b Acta de la XXII reunión del mecanismo de consultas políticas Bolivia-Chile, en Ministerio de Relaciones Exterior, Web: www.minrel.gob.cl/minrel/site/artic/20100715/asocfile/20100715165143/acta_final.pdf

Marín, V.

2007 “Cuarta Época: la República”, en Historia de Bolivia, Ediciones Don Bosco, La Paz, pp. 151-248.
Milet, $\mathrm{P}$.

2004 "Chile-Bolivia: Cien años después". Revista Fuerzas Armadas y Sociedad, Año 18, № 1 y 2, pp. 37-49.

Morales, E.

2011 Discurso del presidente Evo Morales por el Día del Mar, Marzo 23, 2014. DIREMAR, Discursos, Web: www. diremar.gob.bo/node/947

Ovando, A.

1966 Decreto Ley No 7583 del 13 de abril de 1966. Crease el Pabellón de la Fuerza Naval Boliviana. En Derechoteca Bolivia, Web: http://www.derechoteca.com/gacetabolivia/ decreto-ley-7583-del-13-abril-1966/

Ovando, $\mathrm{C}$.

2015 "La noción de soberanía en las propuestas bolivianas para la salida al mar: el caso Arica Trinacional". Revista Diálogo Andino, $\mathrm{N}^{\circ}$ 48, pp. 127-138.

Pérez, E.

2004 Una misión. Las Trampas de la relación chilenoboliviana. Debate, Santiago de Chile.

República de Bolivia

1922 Ley de 25 de Marzo. Extraído: http://www.lexivox.org/ norms/BO-L-19220325.xhtml

República de Bolivia

2011 Decreto Supremo No 0834 . DIREMAR, Marco normativo. En Biblioteca Virtual de la Cooperación Alemana, Extraído: http://www.bivica.org/upload/decreto-supremo_834.pdf

República de Bolivia

2013 Application Instituting proceedings. Obligation to negotiate access to the Pacific Ocean, Extraído: https:// www.icj-cij.org/files/case-related/153/17338.pdf

República de Chile

1866 Tratado de límites entre la República de Chile i la de Bolivia. Extraído: www.leychile.cl/N?i=220184\&f=1866-12-13\&p

República de Chile

2016 Application Instituting proceedings. Dispute over the Status and Use of the Waters of the Silala, Extraído: https:// www.icj-cij.org/files/case-related/162/162-20160606-APP01-00-EN.pdf

Rivas, P. y Sepúlveda, D.

2011 "Instituciones de América del Sur: Entre la integración y lo heterogéneo". Revista Escenarios Actuales, año 16, $\mathrm{N}^{\circ}$ 2, septiembre, pp. 17-24.

Valencia, A.

1982 "Capítulo XXV: costa marítima para el desarrollo vital de Bolivia”. En Geopolítica del Litoral Boliviano, Editorial Juventud, La Paz, Bolivia, pp. 519-538. 